\title{
Comparison of laparoscopic versus open gastrectomy with D2 lymph node dissection for advanced gastric cancer: A meta-analysis
}

Sandrie Mariella Mac, PhD ; Ashish Bahadur Malla, MS

${ }^{1}$ Department of Gastrointestinal Surgery, The First Affiliated Hospital of Chongqing Medical University, China

${ }^{2}$ Department of Gastrointestinal Surgery, Grande International Hospital, Kathmandu, Nepal

Corresponding author

Ashish B Malla, MS

Email: ashirc88@gmai.com

Received 22 Feb 2019

Accepted 3 June 2019

\begin{abstract}
For many decades, D2 procedure has been accepted in the far-east as the standard treatment for both early (EGC) and advanced gastric cancer (AGC). In case of AGC, the debate on the extent of nodal dissection has been open for many years in order to highlight the safety and efficacy of treatment, hence this study.
\end{abstract}

A comprehensive literature research was performed in PubMed to identify studies that compared laparoscopic- assisted gastrectomy (LAG) and open gastrectomy (OG) with D2 lymph node dissection (D2-LND) for treatment of AGC for the last five years. Data of interest were checked and subjected to meta-analysis with RevMan 5.3 software. The pooled risk ratios (RR) and weighted mean difference (WMD) with 95\% confidence intervals (CI) were calculated.

Overall, 19 studies were included in this meta-analysis. LG had some advantages over OG, including shorter hospitalization (WMD -2.31; $95 \% \mathrm{Cl}-4.09$ to $-0.53 ; \mathrm{P}=0.01$ ), less blood loss (WMD -120.49; $95 \% \mathrm{Cl}-174.27$ to $-66.71 ; \mathrm{P}<0.01$ ), faster bowel recovery (WMD $-0.55 ; 95 \% \mathrm{Cl}-0.86$ to $-0.24 ; \mathrm{P}$ $<0.01$ ) and earlier ambulation (WMD $-0.75 ; 95 \% \mathrm{Cl}-1.38$ to $-0.11 ; \mathrm{P}=0.02$ ). In terms of surgical and oncological safety, LG could achieve similar lymph nodes (WMD, $-0.94,95 \% \mathrm{Cl},-2.95$ to 1.06 ; $\mathrm{P}=0.36$ ), a lower complication rate [odds ratio $(\mathrm{OR})=0.80 ; 95 \% \mathrm{Cl}, 0.68-0.97 ; \mathrm{P}=0.02$ ], and overall survival (OS) and disease-free survival (DFS) comparable to OG.

In conclusion, for AGCs both techniques (LAG and OG) appeared comparable in short- and longterm results. More time was needed to perform LAG; nonetheless, it had some advantages in achieving faster postoperative recovery over OG. In order to clarify this controversial issue ongoing trials and future studies are needed.

Keywords: Advanced gastric cancer, Laparoscopic gastrectomy, Open gastrectomy, D2 lymph node dissection, meta-analysis

\section{Introduction}

With the third highest rate of mortality, cancer of the stomach is one of the most malignant tumors in the world ${ }^{1}$. In fact, around three-quarters of all new cases and deaths occur in Eastern Asia, Eastern Europe and South America, and almost close to half (42\%) in China ${ }^{2}$. Despite considerable progress in the diagnosis and treatment of gastric cancer, it remains a major health problem and requires a multidisciplinary approach in which surgery is the cornerstone in the treatment of gastric cancer ${ }^{3}$, which includes conventional open gastrectomy (OG) and laparoscopy-assisted gastrectomy (LAG).

For a long time in history, conventional open gastrectomy (OG) surgery remains the treatment of choice for gastric cancer. A complete resection along with lymph node dissection was recognized as broad as the only one cure for gastric cancer ${ }^{4}$. 
But since its first description in 1991 by Kitano ${ }^{5}$, laparoscopic gastrectomy (LG) has experienced rapid development and has been widely accepted and widely used to treat EGC nowadays, especially in East Asia( China, Japan and Korea) ${ }^{6,7}$.

With curative intent, the D2 lymph node dissection has been widely applied in traditional open surgery for locally advanced gastric cancer $(A G C)^{8}$, however, the use of this procedure in LG for AGC is discussed. The oncological safety of LG in AGC treatment has not been well evaluated, and it remains to be confirmed whether laparoscopic surgery can still guarantee the advantage of minimal invasion, whether it increases perioperative complications and mortality, and whether it can achieve the same degree of radicalism as open surgery. To this end, we conducted a meta-analysis by comparing LAG with OG with D2 lymph node dissection for AGC with regard to their short- and long-term outcomes in order to determine the current status of LAG.

\section{Materials \& Methods}

\section{Search strategy:}

A comprehensive PubMed search from January 2011 to February 2016 was performed using the following Mesh search headings and text words: "laparoscopy-assisted gastrectomy", "laparoscopicassisted gastrectomy", "open gastrectomy", "conventional gastrectomy", "gastric cancer", "gastric carcinoma", "advanced gastric cancer", "D2 dissection". Logical combinations of these and related terms (stomach, neoplasm) were used to maximize sensitivity and only studies published in English were included. Title and abstracts of each identified publication were screened, and only publications that reported the full texts for the clinical outcomes of this analysis were further retrieved. Reference lists of systematic reviews or meta-analysis were additionally checked to identify potential eligible studies.

\section{Study eligibility:}

Studies following below criteria were selected:

(1) RCTs and nonrandomized comparative studies

(2) Patients with advanced gastric carcinoma and/or with locally advanced were acceptable, without limitations for race, age or gender and where the patients in the LG and OG groups were compared; and the staging system was based on the individual reports
(3) Studies comparing the short and long-term outcomes of LADG and OG with D2 LND.

Exclusion criteria included:

(1) robot-assisted gastrectomy

(2) no OG as a control

(3) abstract only

(4) recurrent gastric cancer or palliative resection cases

(5) duplicate publication or publications with sufficient data.

\section{Data extraction:}

EndNote (Version X7, Thomson Reuters) was used to merge the search results and remove duplicate records of the same report. Titles and abstracts were screened. Articles deemed potential for inclusion were reviewed by two reviewers independently and data extracted in predefined forms. Disagreement was resolved by discussion with the two other reviewers. Relevant data included: first author, year of publication; geographical region; study period; type of study; sample size for each technique, laparoscopic technique. The short-term outcomes included operation time, blood loss, harvested lymph nodes, tumor size, first flatus time, oral diet time (soft or liquid), post-operative hospital stay, ambulation, morbidity (or the incidence of postoperative complications), mortality (here defined as hospital mortality). Postoperative complications were classified as surgical complications and medical complications. The long-term outcomes included cancer recurrence and survival rate.

\section{Quality assessment:}

For all the included studies ${ }^{9}$, to assess their quality we used the modified Newcastle-Ottawa Scale. The total score was 9 stars, and the quality of each study was graded as level 1 (0-5 stars) or level 2 (6-9 stars). Studies $\geq 5$ stars were considered as highquality studies.

\section{Data synthesis and statistical analysis:}

The "Review Manager 5.3" software from the Cochrane collaboration was used for statistical analysis. For all statistical calculations, RRs was used for dichotomous variables and WMDs for continuous variables with a $95 \%$ confidence interval (CI). For this latter, data in the form of means and standard deviation allowed statistical analysis. A 
random-effect model was used to avoid statistical heterogeneity between the studies owing to the high heterogeneity of the studies; otherwise, fixed-effects model was used. Heterogeneity was calculated by $\mathrm{Chi}^{2}$ and the $\mathrm{I}^{2}$ test. The fixed effect model was used if no significant heterogeneity was observed among the included studies $(P>$ $0.1, \mathrm{I}^{2}<50 \%$ ). Publication bias was evaluated by a funnel plot. For all analyses, $\mathrm{P} \leq 0.05$ was considered statistically significant. For dichotomous outcomes, the Mantel-Haenszel method was used for both fixed effect analysis and random effect (DerSimonian) $)^{10}$ analysis. For continuous data, an inverse variance method was used.

\section{Results}

A total of 19 studies $^{11-29}$ that included 4949 (2534 in LAG and 24155 in OG) gastrectomy with D2 lymph node resection for $A G C$ were considered eligible for our meta-analysis. For every study, analysis of the demographics and clinic-pathological characteristics of patients treated by LG and OG did not differ from each other. Among the 19 articles, twelve studies originated from China ${ }^{11-22}$, three from Korea ${ }^{23-25}$, three from Japan ${ }^{26-28}$, and one from Italy ${ }^{29}$. Selected studies were published within 5 years, from 2011 to 2016, and case numbers varied from 18 to 1078 . Proximal, distal, total and subtotal gastrectomies were used as surgical options. Gastrointestinal tract reconstruction modalities included Billroth-I/ II, Roux-en-Y anastomosis, eosophago-gastrostomy, and eosophago-jejunostomy. None of the studies had reported the use of neo-adjuvant chemoradiotherapy, but one study ${ }^{28}$ reported the use of chemo-radiotherapy in postoperative period. The follow-up ranged from 30 days to 5 years. Detailed information on study characteristics is shown in Table 1 and quality assessment in Table 2, all study had a score of $\geq 6$ stars.

The quality of each study was graded as low level (total score 0-5) or high level (total score 6-9).

a) Selection: (1) Assignment for treatment: One star was assigned if details of criteria for assignment of patients to treatments provided. (2) One star was assigned if the laparoscopic-assisted distal gastrectomy group was representative of patients for gastric cancer; no star was assigned if groups of patients were selected or selection of the group was not described. (3) One star was assigned if the open distal gastrectomy group was representative of patients for gastric cancer; no star was assigned if groups of patients were selected or selection of the group was not described.

b) Comparability: Comparability variables were as follows: 1 , age; 2 , sex; 3 , depth of tumor invasion on preoperative diagnosis; 4 , extent of lymphadenectomy; 5 , median or mean followup; 6, American Society of Anesthesiologists status; 7, tumor size; 8, postoperative pathologic stage; and 9, histological type. (4)

Table 1: Bas eline characteristics of studies included in the meta-analysis

\begin{tabular}{|c|c|c|c|c|c|c|c|c|c|c|c|}
\hline References & Country & $\begin{array}{c}\text { Publication } \\
\text { year }\end{array}$ & Study period & $\begin{array}{l}\text { Study } \\
\text { design }\end{array}$ & Total & $\begin{array}{l}\text { Patients } \\
\text { LADG }\end{array}$ & $O G$ & $\begin{array}{l}\text { Tumor stages of } \\
\text { AGCs }\end{array}$ & $\begin{array}{c}\text { Surgical } \\
\text { extension }\end{array}$ & $\begin{array}{l}\text { Construction } \\
\text { methods }\end{array}$ & $\begin{array}{l}\text { Median } \\
\text { Follow-up }\end{array}$ \\
\hline Caietal [11] & China & 2011 & $2008 / 03-2009 / 12$ & $\mathrm{RCT}$ & 96 & 49 & 47 & Ib-IIIb & PGDG,TG & B-I/II & 2.1 months \\
\hline Jeong et al [23] & Korea & 2011 & $2005 / 01-2007 / 12$ & Retro & 185 & 109 & 76 & $\mathrm{Ib}-\mathrm{IV}$ & DG,TG & B-III, R-Y & 36.8 months \\
\hline Scatizietal [29] & Italy & 2011 & $2006 / 01-200909$ & Retro & 60 & 30 & 30 & II-IIIb & SG & R-Y & 18 months \\
\hline Shrang et al. [12] & China & 2011 & $2005 / 08-2007 / 12$ & Retro & 70 & 35 & 35 & $\mathrm{Ib}-\mathrm{IIIb}$ & DG & B-II & 35 months \\
\hline Chen et al [13] & China & 2012 & $2008 / 01-201012$ & Retro & 336 & 224 & 112 & $\mathrm{Ib}-\mathrm{IIIb}$ & DG,TG & B-III.R-Y & 19 months \\
\hline Chưn et al [24] & Korea & 2012 & $2004 / 01-2009 / 12$ & Retro & 119 & 52 & 67 & $\mathrm{Ib}$-IIla & DG & B-III, R-Y & 5 years \\
\hline Hamabe et al [26] & Japan & 2012 & $2000 / 01-2009112$ & Retro & 167 & 66 & 101 & $\mathrm{Ib}-\mathrm{IIIb}$ & DGTG & B-III.R-Y & 5 years \\
\hline Kimetal [25] & Korea & 2012 & $1999 / 08-2007 / 06$ & Retro & 176 & 88 & 88 & $\mathrm{Ib}$-IIIc & PGDGSG & B-IIII,R-Y,EG & 5 years \\
\hline Shinohara et al [27] & Japan & 2013 & $1997 / 10-2008 / 12$ & Retro & 309 & 186 & 123 & $\mathrm{cT2}-\mathrm{T} 4$ & PGDG,TG & B-I, R-Y & 48.8 months \\
\hline Lin et al [14] & China & 2013 & $2008 / 01-201012$ & Retro & 166 & 83 & 83 & $\mathrm{Ib}-\mathrm{IIIb}$ & DG,TG & B-III,R-Y & 23 months \\
\hline Bo etal [15] & China & 2013 & $2004 / 01-201012$ & Retro & 234 & 117 & 117 & $\mathrm{Ib}-\mathrm{IIIb}$ & TG & R-Y & 61.2 months \\
\hline Fang et a1 [16] & China & 2013 & $2009 / 08-2011 / 01$ & Retro & 112 & 50 & 62 & $\mathrm{NR}$ & $\mathrm{NR}$ & B-I/II & 18 months \\
\hline Yamanaka et al [28] & Japan & 2013 & $2000 / 01-201012$ & Retro & 18 & 9 & 9 & IV & DG,TG & $\mathrm{NR}$ & 20.5 months \\
\hline Lietal $[17]$ & China & 2013 & $2009 / 03-2011 / 06$ & Retro & 239 & 106 & 133 & $\mathrm{Ib}$-IIIc & DG,TG & B-III.R-Y & 15 months \\
\hline Fang etal [18] & China & 2014 & $2005 / 042009 / 10$ & Retro & 174 & 87 & 87 & $\mathrm{Ib}-\mathrm{IIIC}$ & DG,TG & EJ,GI & 44 months \\
\hline Zhang et al. [19] & China & 2015 & $2007 / 01-201412$ & Retro & 172 & 86 & 86 & Ib-IIIc & NR & $\mathrm{NR}$ & 39 months \\
\hline Lietal $[20]$ & China & 2016 & $2012 / 04201412$ & Retro & 202 & 101 & 101 & II-IIIb & DG,TG & $\mathrm{NR}$ & $\mathrm{NR}$ \\
\hline Hu et al. [21] & China & 2016 & $2012 / 09-201412$ & Retro & 1036 & 517 & 519 & $\mathrm{Ib}-\mathrm{Iv}$ & $\mathrm{DG}, \mathrm{TG}$ & B-III, R-Y & 30 days \\
\hline Lin et al [22] & China & 2016 & $2005 / 01-2011 / 12$ & Retro & 1078 & 539 & 539 & $\mathrm{Ib}-\mathrm{IIIC}$ & DG,TG & $\mathrm{NR}$ & 45 months \\
\hline
\end{tabular}

$N R$ not reported, $D G$ distal gastrectomy, $R C T$ randomized controlled trial Retro retrospective, $P G$ proximal gastrectomy, $T G$ toal gastrectomy, $S G$ subtotal gastrectomy, Bil Billroth-I, B-ll Billroth II, R-Y Roux-ent Y, EG eosophagogastrostomy, EJ eosophagojejunostonty 
Table 2: Quality assessment of included studies

\begin{tabular}{|c|c|c|c|c|c|c|c|c|}
\hline \multirow[t]{2}{*}{ Reference } & \multicolumn{3}{|c|}{ Selection } & \multicolumn{2}{|c|}{$\begin{array}{c}\text { Comparability of } \\
\text { group }\end{array}$} & \multicolumn{2}{|c|}{ Outcomes } & \multirow[t]{2}{*}{ Total } \\
\hline & 1 & 2 & 3 & 4 & 5 & 6 & 7 & \\
\hline Cai et al. & $*$ & $*$ & $*$ & $* *$ & $*$ & $*$ & & $* * * * * * *$ \\
\hline Jeong et al. & $*$ & $*$ & $*$ & $*$ & $*$ & $*$ & & $* * * * * *$ \\
\hline Scatizzi et al. & $*$ & $*$ & $*$ & $* *$ & $*$ & $*$ & $*$ & $* * * * * * * *$ \\
\hline Shuang et al. & $*$ & $*$ & $*$ & $* *$ & & $*$ & $*$ & $* * * * * * *$ \\
\hline Chen et al. & $*$ & $*$ & $*$ & $* *$ & $* *$ & $*$ & $*$ & $* * * * * * * * *$ \\
\hline Chun et al. & $*$ & $*$ & $*$ & $* *$ & $*$ & $*$ & $*$ & $* * * * * * * *$ \\
\hline Hamabe et al. & $*$ & $*$ & $*$ & $* *$ & $*$ & & $*$ & $* * * * * * *$ \\
\hline Kim et al. & $*$ & $*$ & $*$ & $* *$ & $*$ & & $*$ & $* * * * * *$ \\
\hline Shinohara et al. & $*$ & $*$ & $*$ & $* *$ & $* *$ & $*$ & $*$ & $* * * * * * * * *$ \\
\hline Lin et al. & $*$ & $*$ & $*$ & $* *$ & $*$ & $*$ & $*$ & $* * * * * * * *$ \\
\hline Bo et al. & $*$ & $*$ & $*$ & $* *$ & $*$ & $*$ & & $* * * * * * *$ \\
\hline Fang et al. & $*$ & $*$ & $*$ & $* *$ & $*$ & $*$ & & $* * * * * * * *$ \\
\hline Yamanaka et al. & $*$ & $*$ & $*$ & $* *$ & $*$ & $*$ & & $* * * * * * *$ \\
\hline Li et al & $*$ & $*$ & $*$ & $* *$ & $*$ & & $*$ & $* * * * * * *$ \\
\hline Fang et al. & $*$ & $*$ & $*$ & $* *$ & $* *$ & & $*$ & $* * * * * * * *$ \\
\hline Zhang et al. & $*$ & $*$ & $*$ & $*$ & $*$ & & $*$ & $* * * * * *$ \\
\hline Li et al & $*$ & $*$ & $*$ & $*$ & $* *$ & $*$ & $*$ & $* * * * * * * *$ \\
\hline Hu et al. & $*$ & $*$ & $*$ & $* *$ & $* *$ & $*$ & & $* * * * * * * *$ \\
\hline Lin et al. & $*$ & $*$ & $*$ & $* *$ & $* *$ & & $*$ & $* * * * * * * *$ \\
\hline
\end{tabular}

Two stars were assigned if the groups were all comparable for the variables 1-5; 1 star was assigned if one of these five characteristics was not reported, even if there were no other differences between the groups, and other characteristics had been controlled for; and no star was assigned if the two groups differed. (5) Two stars were assigned if the groups were all comparable for the variables 6-9; 1 star was assigned if one of these four characteristics was not reported, even if there were no other differences between the groups, and other characteristics had been controlled for; and no star was assigned if the two groups differed.

c) Outcome assessment: (6) one star was assigned if primary outcome parameters were clearly reported. (7) One star was assigned if more than $90 \%$ of patients were followed up.

\section{1) Comparison of intraoperative effects}

The duration of operation time in LAG group was 59.61 min longer than that in OG group (WMD 59.61; $95 \% \mathrm{Cl} 28.43$, 90.79; $\mathrm{P}<0.01$ ) with the randomeffect model due to significant heterogeneity $\left(I^{2}\right.$
$=100 \%)$. With 12 studies included, the results of estimated blood loss were in favor of LAG group, with a reduction of $120.49 \mathrm{ml}$ than OG (WMD $-120.49 ; 95 \% \mathrm{Cl}-174.27,-66.71 ; \mathrm{P}<0.01)$, same as the patients needed transfusion is less in LAG group compared to the OG (WMD 0.40; 95\% Cl 0.22, 0.72; $P<0.01)$. Concerning the dissected LNs, LAG could achieve the same radical dissection effect as OG (WMD, - $0.94,95 \% \mathrm{Cl},-2.95,1.06 ; \mathrm{P}=0.36$ ) with the random-effect model due to marked heterogeneity $\left(I^{2}=94 \%\right)$ (Figure 1). Furthermore, both procedures yielded comparable proximal (WMD, -0.34, $95 \%$ $\mathrm{Cl},-0.86,0.19 ; \mathrm{P}=0.21$ ), marked heterogeneity $\left(I^{2}=80 \%\right)$ and distal (WMD, $-0.05,95 \% \mathrm{Cl},-0.27$, $0.18 ; \mathrm{P}=0.68$ ) resection margin distance where the level of heterogeneity was not worthy $\left(I^{2}=17 \%\right)$, so the fixed effect model was used. Similar size of tumor was found between LAG group and OG group (WMD $=0.61 ; 95 \% \mathrm{Cl}-5.83,7.05 ; \mathrm{P}=0.85$ ) with the random-effect model due to significant heterogeneity $\left(I^{2}=100 \%\right)$.

Results of analyses of intraoperative effects are shown in Table 3.

LG laparoscopic-assisted gastrectomy, OG open gastrectomy, LN lymph node, WMD weighted mean difference 


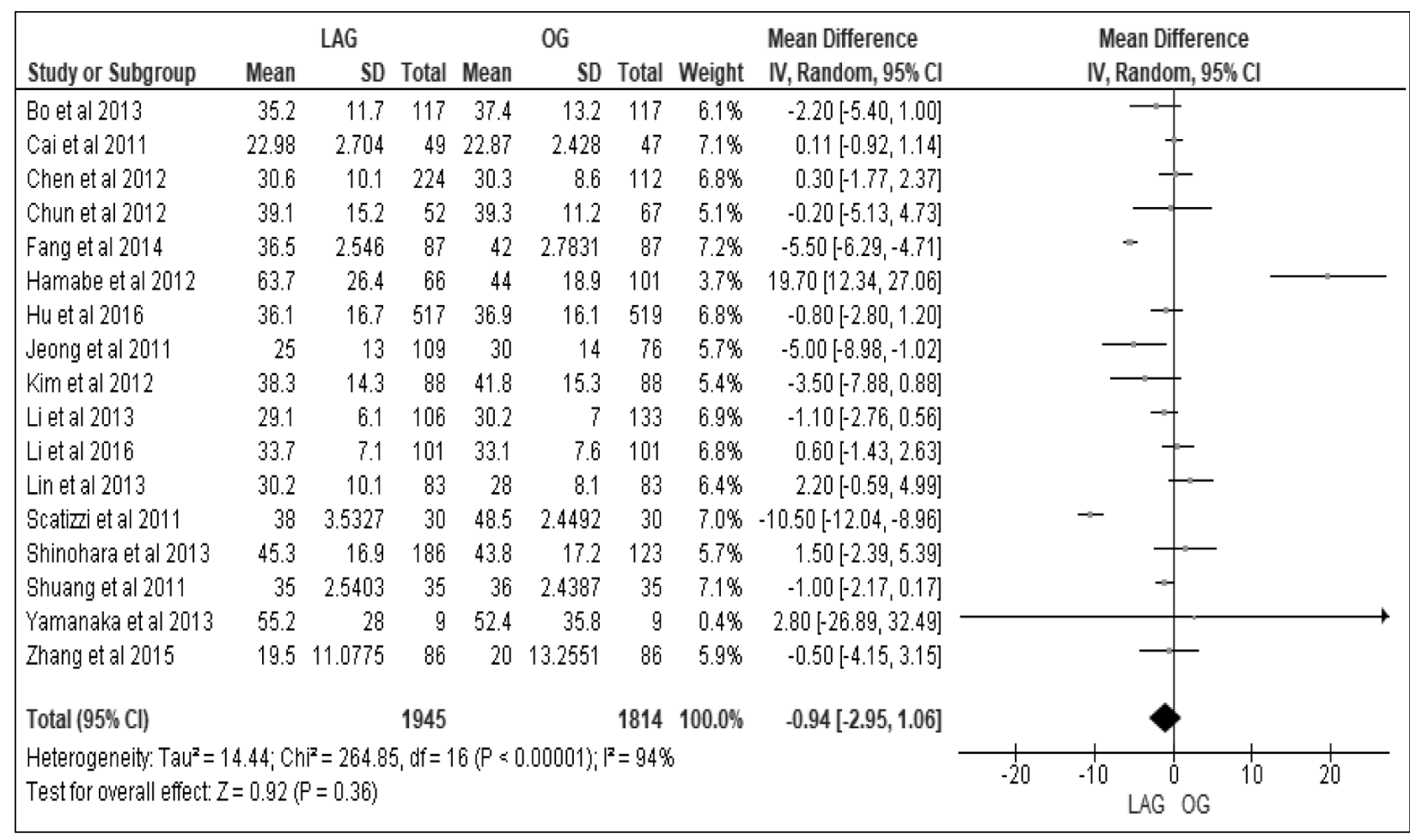

Figure 1: Harvested lymph nodes

Table 3: Comparisons of intraoperative surgical effects

\begin{tabular}{|c|c|c|c|c|c|c|}
\hline \multirow{2}{*}{ Outcomes } & \multirow{2}{*}{$\begin{array}{l}\text { Included } \\
\text { studies }\end{array}$} & \multicolumn{2}{|c|}{ Patients } & \multirow{2}{*}{$\begin{array}{l}\text { Heterogeneity } \\
\qquad\left(I^{2}, P\right)\end{array}$} & \multirow{2}{*}{$\begin{array}{c}\text { Overall effect WMD (95\% } \\
\text { CI) }\end{array}$} & \multirow{2}{*}{$P$ value } \\
\hline & & LAG & OG & & & \\
\hline Operation time, min & 18 & 1995 & 1876 & $100 \%, P<0.01$ & $59.6128 .43,90.79$ & $<0.01$ \\
\hline Estimated blood loss & 12 & 1657 & 1544 & $100 \%, \mathrm{P}<0.01$ & $-120.49-174.27,-66.71$ & $<0.01$ \\
\hline Needed transfusion & 7 & 802 & 681 & $57 \%, 0.03$ & $0.400 .22,0.72$ & $<0.01$ \\
\hline Retrieved LNs & 17 & 1945 & 1814 & $94 \%, \mathrm{P}<0.01$ & $-0.94-2.95,1.06$ & 0.36 \\
\hline Tumor size & 12 & 1719 & 1740 & $100 \%, P<0.01$ & $0.61-5.83,7.05$ & 0.85 \\
\hline $\begin{array}{l}\text { Proximal resection } \\
\text { margin Distance }\end{array}$ & 5 & 883 & 867 & $80 \%, P<0.01$ & $-0.34-0.86,0.19$ & 0.21 \\
\hline $\begin{array}{l}\text { Distal resection margin } \\
\text { Distance }\end{array}$ & 5 & 816 & 812 & $17 \%, 0.31$ & $-0.05-0.27,0.18$ & 0.68 \\
\hline
\end{tabular}

\section{2) Analyses of short-term results}

Laparoscopic surgery has certain advantages compared to open surgery such as less pain, shorter hospitalization, quicker bowel function recovery, earlier resumption of body movement. In agreement with this, we found patients undergoing LAG had: shorter mean time for use of analgesic drugs (WMD -1.87; 95\% Cl -2.57, -1.18; $\mathrm{P}<0.01$ ), shorter hospital stay with 2.31 days (WMD -2.31; $95 \% \mathrm{Cl}-4.09,-0.53 ; \mathrm{P}=0.01$ ). Also, shorter mean time to first flatus with 0.55 day earlier (WMD $-0.55 ; 95 \% \mathrm{Cl}-0.86,-0.24 ; \mathrm{P}<0.01)$, and resumed oral intake much earlier in both soft diet (WMD $-1.60 ; 95 \% \mathrm{Cl}-2.18,-1.01 ; \mathrm{P}<0.01)$ and fluid diet (WMD -0.48; $95 \% \mathrm{Cl}-0.90,-0.06 ; \mathrm{P}=0.03$ ) than those undergoing OG with 1.60 and 0.48 days earlier respectively. In addition, patients treated with LAG needed less time to ambulation than those with OG (WMD $-0.75 ; 95 \% \mathrm{Cl}-1.38,-0.11 ; \mathrm{P}=$ 0.02 ). The perioperative complication rate was also compared. Overall complication rate for LAG was $15 \%$, significantly lower than the rate of $17.6 \%$ of OG (OR, 0.81; 95\% Cl 0.68, 0.97; $\mathrm{P}=0.02$ ) (Figure 2). To be specific, the rates of surgical complications, including leakage, wound infection, bleeding and anastomotic stricture $(\mathrm{OR}, 0.72,95 \% \mathrm{Cl}, 0.55$, $0.94 ; \mathrm{P}=0.02$ ), and medical complications such as respiratory or cardiovascular events, pulmonary embolism, deep venous thrombosis and nonsurgical infections (OR, 0.48, $95 \% \mathrm{Cl}, 0.31,0.74 ; \mathrm{P}$ $=0.001$ ) were both in favor of LAG. Moreover, in 


\begin{tabular}{|c|c|c|c|c|c|c|c|c|c|c|c|}
\hline Study or Subgroup & \multicolumn{2}{|c|}{$\mathrm{LAG}$} & \multicolumn{2}{|c|}{$O G$} & Weight & $\begin{array}{c}\text { Odds Ratio } \\
\text { M-H, Fixed, } 95 \% \mathrm{Cl}\end{array}$ & \multicolumn{3}{|c|}{$\begin{array}{c}\text { Odds Ratio } \\
\text { M-H, Fixed, } 95 \% \mathrm{Cl}\end{array}$} & & \\
\hline Bo et al 2013 & 13 & 117 & 19 & 117 & $6.0 \%$ & $0.64[0.30,1.38]$ & & & - & & \\
\hline Cai et al 2011 & 6 & 49 & 9 & 47 & $2.9 \%$ & $0.59[0.19,1.81]$ & & & & & \\
\hline Chen et al 2012 & 25 & 224 & 17 & 112 & $7.2 \%$ & $0.70[0.36,1.36]$ & & & & & \\
\hline Chun et al 2012 & 5 & 52 & 6 & 67 & $1.7 \%$ & $1.08[0.31,3.76]$ & & & & & \\
\hline Fang et al 2013 & 6 & 50 & 18 & 62 & $5.1 \%$ & $0.33[0.12,0.92]$ & & & & & \\
\hline Fang et al 2014 & 6 & 87 & 5 & 87 & $1.7 \%$ & $1.21[0.36,4.14]$ & & & & & \\
\hline Hamabe et al 2012 & 16 & 66 & 23 & 101 & $4.9 \%$ & $1.09[0.52,2.25]$ & & & & & \\
\hline Hu et al 2016 & 79 & 517 & 67 & 519 & $20.3 \%$ & $1.22[0.86,1.73]$ & & & - & & \\
\hline Jeong et al 2011 & 29 & 109 & 15 & 76 & $4.6 \%$ & $1.47[0.73,2.99]$ & & & & & \\
\hline Kim et al 2012 & 7 & 88 & 7 & 88 & $2.3 \%$ & $1.00[0.34,2.98]$ & & & & & \\
\hline Li et al 2013 & 15 & 106 & 33 & 133 & $9.0 \%$ & $0.50[0.25,0.98]$ & & & & & \\
\hline Li et al 2016 & 22 & 101 & 38 & 101 & $10.6 \%$ & $0.46[0.25,0.86]$ & & & & & \\
\hline Lin et al 2013 & 10 & 83 & 12 & 83 & $3.8 \%$ & $0.81[0.33,1.99]$ & & & & & \\
\hline Scatizi et al 2011 & 2 & 30 & 8 & 30 & $2.7 \%$ & $0.20[0.04,1.02]$ & & & & & \\
\hline Shinohara et al 2013 & 45 & 186 & 35 & 123 & $11.4 \%$ & $0.80[0.48,1.34]$ & & & & & \\
\hline Shuang et al 2011 & 2 & 35 & 3 & 35 & $1.0 \%$ & $0.65[0.10,4.13]$ & & & & & \\
\hline Yamanaka et al 2013 & 1 & 9 & 2 & 9 & $0.6 \%$ & $0.44[0.03,5.93]$ & & & & & \\
\hline Zhang et al 2015 & 9 & 86 & 13 & 86 & $4.2 \%$ & $0.66[0.26,1.63]$ & & & & & \\
\hline Total $(95 \% \mathrm{Cl})$ & & 1995 & & 1876 & $100.0 \%$ & $0.81[0.68,0.97]$ & & & & & \\
\hline Total ewents & 298 & & 330 & & & & & & & & \\
\hline $\begin{array}{l}\text { Heterogeneity: Chi }{ }^{2}=2 \\
\text { Test for overall effect: } 2\end{array}$ & $\begin{aligned} & .54, \mathrm{df}= \\
&= 2.34 \mathrm{P}\end{aligned}$ & $\begin{array}{l}17(\mathrm{P}= \\
=0.02)\end{array}$ & $=0.20) ; 1^{2}=$ & $=21 \%$ & & & $\begin{array}{ll}+0.02 & 0.1\end{array}$ & $\mathrm{LAG}^{1}$ & 1 & 10 & $\frac{1}{50}$ \\
\hline
\end{tabular}

Figure 2: Forest plot of pooled odds ratio of overall complication

terms of mortality, there is no significant differences between the two procedures $(\mathrm{OR}, 0.68,95 \% \mathrm{Cl}$, $0.46,1.02 ; \mathrm{P}=0.06$ ).

The comparison outcomes of short- term results between $L G$ and $O G$ is summarized in Table 4.

LG laparoscopic-assisted gastrectomy, OG open gastrectomy, WMD weighted mean difference, $O R$ odds ratio

\section{3) Analyses of long-term effects}

Seven studies reported data on tumor recurrence, with a moderate heterogeneity observed. The recurrence rate in LAG was $23.45 \%$, which was significantly lower than the rate of $25.23 \%$ in OG (RR, 0.83, $95 \% \mathrm{Cl}, 0.55,1.24 ; \mathrm{P}=0.36$ ) (Figure 3). Thirteen studies ${ }^{13-19,22,24-28}$ reported long-term follow-up ranging from 1 year to 5 years. Five studies $^{13-14,16-17,28}$ reported 1-year OS; two studies ${ }^{16,22}$ reported 3-year OS, and seven ${ }^{15,18-19,24-27}$ reported 5 -year OS. No significant differences were seen in 1-, 3-, or 5-year OS between LAG and OG [1-year: Risk ratio(RR) $=1.02,95 \% \mathrm{Cl}, 0.97-1.08 ; \mathrm{P}=0.42$; 3-year: $\mathrm{RR}=0.97,95 \% \mathrm{Cl}, 0.88-1.07 ; \mathrm{P}=0.53$; 5-year: $\mathrm{RR}=1.08,95 \% \mathrm{Cl}, 1.00-1.16 ; \mathrm{P}=0.04]$ respectively. Regarding RFS, two studies ${ }^{18,26}$ reported 5-year RFS.

Table 4: Comparisons of short-term results

\begin{tabular}{|l|c|c|c|c|c|c|}
\hline \multirow{2}{*}{ Outcomes } & Included & \multicolumn{2}{|c|}{ Patients } & Heterogeneity & Overall effect WMD & \multirow{2}{*}{ P value } \\
\cline { 7 - 8 } & studies & LAG & OG & $(\mathbf{9 5}, \mathbf{P})$ & $\mathbf{C l})$ & \\
\hline Hospital stay & 17 & 1907 & 1788 & $97 \%, \mathrm{P}<0.01$ & $-2.31-4.09,-0.53$ & $<\mathbf{0 . 0 1}$ \\
\hline First flatus & 11 & 1476 & 1373 & $91 \%, \mathrm{P}<0.01$ & $-0.55-0.86,-0.24$ & $<\mathbf{0 . 0 1}$ \\
\hline Oral diet (soft) & 6 & 633 & 458 & $85 \%, \mathrm{P}<0.01$ & $-1.60-2.18,-1.01$ & $<\mathbf{0 . 0 1}$ \\
\hline Fluid intake & 4 & 496 & 409 & $72 \%, \mathrm{P}<0.01$ & $-0.48-0.90,-0.06$ & 0.03 \\
\hline Use of analgesic drugs & 5 & 352 & 352 & $83 \%, \mathrm{P}<0.01$ & $-1.87-2.57,-1.18$ & $<\mathbf{0 . 0 1}$ \\
\hline Ambulation, days & 7 & 1206 & 1031 & $98 \%, \mathrm{P}<0.01$ & $-0.75-1.38,-0.11$ & 0.02 \\
\hline Overal complication & 18 & 1995 & 1876 & $21 \%, 0.20$ & $0.810 .68,0.97$ & 0.02 \\
\hline Surgical complication & 10 & 1014 & 914 & $0 \%, 0.67$ & $0.720 .55,0.94$ & 0.02 \\
\hline Medical complication & 7 & 813 & 663 & $21 \%, 0.27$ & $0.480 .31,0.74$ & 0.001 \\
\hline Mortality & 10 & 1458 & 1345 & $0 \%, 0.49$ & $0.680 .46,1.02$ & 0.06 \\
\hline
\end{tabular}




\begin{tabular}{|c|c|c|c|c|c|c|c|c|c|c|c|}
\hline Study or Subgroup & $\begin{array}{r}\text { LAG } \\
\text { Events }\end{array}$ & Total & $\begin{array}{r}\text { OG } \\
\text { Events }\end{array}$ & Total & Weight & $\begin{array}{c}\text { Risk Ratio } \\
\text { M-H, Random, } 95 \% \mathrm{Cl}\end{array}$ & \multicolumn{5}{|c|}{$\begin{array}{c}\text { Risk Ratio } \\
\text { M-H, Random, } 95 \% \mathrm{Cl}\end{array}$} \\
\hline Chun et al 2012 & 4 & 52 & 7 & 67 & $8.1 \%$ & $0.74[0.23,2.38]$ & & & - & & \\
\hline Fang et al 2014 & 36 & 87 & 45 & 87 & $20.8 \%$ & $0.80[0.58,1.10]$ & & & & & \\
\hline Hamabe et al 2012 & 4 & 66 & 22 & 101 & $9.7 \%$ & $0.28[0.10,0.77]$ & & & & & \\
\hline Jeong et al 2011 & 26 & 109 & 28 & 76 & $18.6 \%$ & $0.65[0.41,1.01]$ & & & & & \\
\hline Kim et al 2012 & 13 & 88 & 15 & 88 & $14.4 \%$ & $0.87[0.44,1.71]$ & & & - & & \\
\hline Shinohara et al 2013 & 53 & 186 & 17 & 123 & $17.7 \%$ & $2.06[1.25,3.39]$ & & & $\rightarrow-$ & & \\
\hline Yamanaka et al 2013 & 4 & 9 & 5 & 9 & $10.7 \%$ & $0.80[0.31,2.04]$ & & & & & \\
\hline Total (95\% Cl) & & 597 & & 551 & $100.0 \%$ & $0.83[0.55,1.24]$ & & & & & \\
\hline Total events & 140 & & 139 & & & & & & & & \\
\hline $\begin{array}{l}\text { Heterogeneity: Tau }{ }^{2}= \\
\text { Test for overall effect: }\end{array}$ & $\begin{array}{l}18 ; \mathrm{Chi}^{2}= \\
=0.92(\mathrm{P}\end{array}$ & $\begin{array}{l}=18.51, \\
=0.36)\end{array}$ & $d f=6(P$ & $=0.00$ & $55)_{1}^{2}=68$ & & 0.02 & 0.1 & OG & 10 & $\frac{1}{50}$ \\
\hline
\end{tabular}

Figure 3: Recurrence rate.

The 5-year RFS of LAG and OG were $53.6 \%$ and $34.6 \%$ respectively ( $R R=1.67,95 \% \mathrm{Cl}, 1.32-2.11$; $P<0.01$ ). About DFS, three studies ${ }^{19,25,27}$ reported 5-year DFS. The 5-year DFS of LAG and OG were $66.66 \%$ and $62.62 \%$ respectively ( $R R=1.07,95 \%$ $\mathrm{Cl}, 0.96-1.19 ; \mathrm{P}=0.25)$.

Results of meta-analysis of long-term effects are presented in Table 5.

Table 5: Comparisons of long-term results short-term outcomes in AGC patients ${ }^{33,34}$. It is for this reason that we decided to conduct this metaanalysis in order to shed more light on the value of the LAG with D2 LND for AGC.

In our study, we can observe that LAG demonstrated several advantages for AGC treatment. LAG group patients showed significantly less blood loss, faster recovery, earlier ambulation and shorter hospital stay compared to those treated by OG.

\begin{tabular}{|l|c|c|c|c|c|c|}
\hline \multirow{2}{*}{ Outcomes } & \multirow{2}{*}{$\begin{array}{c}\text { Included } \\
\text { studies }\end{array}$} & \multicolumn{2}{|c|}{ Patients } & \multirow{2}{*}{$\begin{array}{c}\text { Heterogeneity } \\
\left(\mathbf{I}^{\mathbf{2}}, \mathbf{P}\right)\end{array}$} & $\begin{array}{c}\text { Overall effect RR } \\
\mathbf{( 9 5 \%} \mathbf{C I})\end{array}$ & P-value \\
\hline Recurrence & 7 & 597 & 551 & $68 \%, 0.36$ & $0.83,0.55-1.24$ & 0.005 \\
\hline 1-year OS & 5 & 472 & 399 & $20 \%, 0.29$ & $1.02,0.97-1.08$ & 0.42 \\
\hline 3-year OS & 2 & 589 & 601 & $0 \%, 0.97$ & $0.97,0.88-1.07$ & 0.53 \\
\hline 5-year OS & 7 & 682 & 669 & $0 \%, 0.47$ & $1.08,1.00-1.16$ & 0.04 \\
\hline 5-year RFS & 2 & 153 & 188 & $0 \%, 0.53$ & $1.67,1.32-2.11$ & $<0.01$ \\
\hline 5-year DFS & 3 & 360 & 297 & $0 \%, 0.88$ & $1.07,0.96-1.19$ & 0.25 \\
\hline
\end{tabular}

LG laparoscopic gastrectomy, OG open gastrectomy, OS overall survival, RFS recurrence-free survival, DFS disease-free survival, RR risk ratio

\section{Discussion}

Important advantages, such as less blood loss, less pain, faster postoperative recovery, reduced hospital stay and early return to normal bowel function are offered by laparoscopic resection over open surgical procedures for patients with gastric cancer ${ }^{30,31}$. Laparoscopic D2 LND for AGC was first reported by Uyama et al. ${ }^{32}$ in 2000 . While the technical feasibility remains controversial, Desiderio et al. described the application of minimally invasive surgery (MIS) regarding the
These advantages are consistent with the findings of fast track surgery and benefits to AGC patient's recovery ${ }^{35,36}$. Less blood loss found in LAG group is same as for scholars who found that it could reduce the risk of adverse effects such as acute lung injury, hypothermia ${ }^{37}$. Faster bowel function recovery and shorter postoperative hospital findings are identical with previous meta-analysis done by Ding, Wang, Qiu and Huang ${ }^{38-41}$.

We also found in this study that the operation time for LAG is longer, explainable by the skill and the familiarity of surgeons with the laparoscopic system influencing the length of operation time. Some studies reporting the learning curve of LAG 
in AGCs claimed that LG could be done as quickly as OG, if and only if, experienced surgeons, likely about after 40 cases, do it the operation time could reach a plateau ${ }^{42-46}$. Another important finding was the surgical and oncological safeties of LAG. The latter were comparable with or even superior to those of OG, thus, supporting the application of LAG with D2 LND for AGC.

In this study, we found that both, overall and specific complication rates in LAG groups were much lower. As we already know, LG has the inherent benefit of minimal invasiveness, reducing the risk of causing massive tissue and organ damage during an operation, therefore, would lead to fewer complications (15\% in LAG group versus $17.6 \%$ in OG group). Similarly, compared to OG group, we found fewer medical complications in LAG group - medical complications such as respiratory and cardiovascular events, pulmonary embolism, deep venous thrombosis and non-surgical infections are potential life threatening events. Here, we found a rate of $4.67 \%$ medical complication for LG patients, which is significantly lower compared to $8.9 \%$ in OG group. This difference could also be attributed to the benefits of laparoscopic surgery as, not only the LG patients had lesser hospital stay and quicker recovery, but also lesser probability to acquire a nosocomial infection ${ }^{47}$; also these sunsets of patients were capable of early mobilization/ambulation than OG patients, thereby reducing the risk of developing hypostasis and deep venous thrombosis ${ }^{48}$.

From the oncology security standpoint, LG is comparable to OG. In fact, at specific stages of the disease, an adequate LN dissection is very important for prognosis in the treatment of gastric cancers not only to reduce the possibility of recurrence and metastasis ${ }^{49}$, but also renders survival benefits ${ }^{50}$. As D2 Lymphadenectomy is already considered as a method of choice for treating AGC in East Asia ${ }^{51}$, it leads us to believe that the success of this practice is an essential part of the radical resection for the AGC treatment ${ }^{52}$. And as we found in our metaanalysis, the number of LNs retrieved in LG with D2 lymphadenectomy did not differ much from that observed in the OG suggesting that LG had lymphadenectomy efficiency comparable to that of OG, which matched with Ding's ${ }^{38}$ Wang's ${ }^{39}$ and Quan's ${ }^{53}$ meta-analysis.

Another variable that also affects the oncological results is resection margin distance. It is well established that the main objective of radical resection is complete removal of tumor mass and that, in many cancers, a close correlation exists between positive resection margin and increased risk of local recurrence and consequently, decreased OS and DFS ${ }^{54}$. As suggested by recent studies, the status of surgical margin could be considered as an independent prognostic factor for GC patients ${ }^{55,56}$ thereby ensuring complete resection of the tumor tissues by a sufficient distance between the edge of the tumor and the resection margin, and also reduced the risk of a positive resection margin ${ }^{57}$. Thus it may in part reflect the possibility of healing of surgical procedures in the evaluation of the distance of the resection margin. Here, we found that there is no difference in the distance between the proximal and distal resection margin for the two techniques, suggesting that both, LAG and OG, possessed comparable curability and oncological safety. Deng et al. ${ }^{58}$ suggested in their study that the size of the tumor should also be considered an important clinic pathology factor in order to improve the accuracy of the prediction of the prognosis of the GC patients. When compared to Quan's meta-analysis ${ }^{53}$, we observed that the tumor size in OG was much larger than in LG. There is significant heterogeneity among the articles in terms of tumor sizes.

For long-term results, in order to evaluate the direct effects of surgical interventions, OS, DFS and recurrence were used. Our study showed that different types of survival seemed almost the same for LG as for OG. The results for 1-, 3-, and 5-year OS and 5-year RFS and 5-year DFS were separately compared, showing that the effects of treatment with LAG were no comparable to OG. Unlike Chen et al. analysis ${ }^{59}$, recurrences observed in LG Group was less, which apparently corresponded to the studies performed by Huang ${ }^{41}$ and Quan ${ }^{53}$ and we theorize that this variation in finding is relative to the nature of the studies included for the analysis. In order to broaden the case pool and eliminate confounding bias at the same time, only recently published articles were included and rejected those where EGC and AGC were treated together.

And as for the interpretation of the conclusions of this study, we do not overlook certain limitations which are as follow. Firstly, only one RCT was included in our meta-analysis and most of the studies were carried out retrospectively. Secondly, ranging from a total of 8 to 1078 patients, the case volumes of the selected studies varied greatly. In 
such cases, the experience of surgeons would greatly influence the comparisons of surgical results, leading to a misinterpretation of the results of surgical procedures, and explains the high heterogeneity among the studies. This scenario necessitates future trials with prospective design and multi-center participation.

\section{Conclusions}

This study has allowed us to demonstrate that in both short- and long-term, LG with D2 LND could be as effective as OG to treat AGC patients. It has also been shown that LG had several advantages such as minimal invasion, faster recovery and shorter hospitalization. Currently, there are ongoing RCTs studying the value of LG over OG, but in the waiting of the publication of their findings, and based on our analysis, as well as the previous meta-analyses, we can conclude that LG may very well be applied for the treatment of AGC, especially if experienced surgeons perform it.

\section{Acknowledgements}

We acknowledge all authors whose publications could be included in our meta-analyses.

\section{References}

1. Ferlay J, Soerjomataram I, Dikshit R, Eser S, Mathers C, Rebelo M, et al. Cancer incidence and mortality worldwide: sources, methods and major patterns in GLOBOCAN 2012. Int J Cancer. 2015;136(5):E359-86.

2. Lin $Y$, Ueda J, Kikuchi S, Totsuka $Y$, Wei WQ, Qiao YL, et al. Comparative epidemiology of gastric cancer between Japan and China. World J Gastroenterol. 2011;17(39):4421-8.

3. Li QG, Li P, Tang D, Chen J, Wang DR. Impact of postoperative complications on long-term survival after radical resection for gastric cancer. World J Gastroenterol. 2013;19(25):4060-5.

4. Shin D, Park SS. Clinical importance and surgical decision-making regarding proximal resection margin for gastric cancer. World J Gastrointest Oncol. 2013;5(1):4-11.

5. Kitano S, Iso $\mathrm{Y}$, Moriyama M, Sugimachi K. Laparoscopy-assisted Billroth I gastrectomy. Surg Laparosc Endosc. 1994;4(2):146-8.
6. Kim $\mathrm{HH}$, Han SU, Kim MC, Hyung WJ, Kim $W$, Lee $\mathrm{HJ}$, et al. Prospective randomized controlled trial (phase III) to comparing laparoscopic distal gastrectomy with open distal gastrectomy for gastric adenocarcinoma (KLASS 01). J Korean Surg Soc. 2013;84(2):123-30.

7. Nakamura K, Katai H, Mizusawa J, Yoshikawa $\mathrm{T}$, Ando $\mathrm{M}$, Terashima $\mathrm{M}$, et al. A phase III study of laparoscopy-assisted versus open distal gastrectomy with nodal dissection for clinical stage IA/IB gastric Cancer (JCOG0912). Jpn J Clin Oncol. 2013;43(3):324-7.

8. Song KY, Kim SN, Park CH. Laparoscopyassisted distal gastrectomy with D2 lymph node dissection for gastric cancer: technical and oncologic aspects. Surg Endosc. 2008;22(3):655-9.

9. Panesar SS, Athanasiou T, Nair S, Rao C, Jones C, Nicolaou $\mathrm{M}$, et al. Early outcomes in the elderly: a meta-analysis of 4921 patients undergoing coronary artery bypass grafting-comparison between off-pump and on-pump techniques. Heart. 2006;92(12):1808-16.

10. DerSimonian R, Laird N. Meta-analysis in clinical trials. Control Clin Trials. 1986;7(3):177-88.

11. Cai J, Wei D, Gao CF, Zhang CS, Zhang H, Zhao T. A prospective randomized study comparing open versus laparoscopy-assisted D2 radical gastrectomy in advanced gastric cancer. Dig surg. 2011;28(5-6):331-7.

12. Shuang J, Qi S, Zheng J, Zhao Q, Li J, Kang Z, et al. A case-control study of laparoscopyassisted and open distal gastrectomy for advanced gastric cancer. J Gastrointest Surg. 2011;15(1):57-62.

13. Chen QY, Huang CM, Lin JX, Zheng CH, Li P, Xie JW, et al. Laparoscopy-assisted versus open D2 radical gastrectomy for advanced gastric cancer without serosal invasion: a case control study. World J Surg Oncol. 2012;10:248.

14. Lin JX, Huang $\mathrm{CM}$, Zheng $\mathrm{CH}$, Li $\mathrm{P}$, Xie JW, Wang JB, et al. Laparoscopy-assisted gastrectomy with D2 lymph node dissection for advanced gastric cancer without serosa invasion: a matched cohort study from South China. World J Surg Oncol. 2013;11:4. 
15. Bo T, Peiwu Y, Feng Q, Yongliang Z, Yan S, Yingxue $\mathrm{H}$, et al. Laparoscopy-assisted vs. open total gastrectomy for advanced gastric cancer: long-term outcomes and technical aspects of a case-control study. J Gastrointest Surg. 2013;17(7):1202-8.

16. Fang F, Han F, Ding YL, Wang HJ. Comparison of laparoscopy-assisted surgery and laparotomy for treating locally advanced distal gastric antral cancer. Exp Ther Med. 2013;6(3):753-8.

17. LiZX, XuYC, Lin WL, Chen J, Wu HY. Therapeutic effect of laparoscopy-assisted D2 radical gastrectomy in 106 patients with advanced gastric cancer. J BUON. 2013;18(3):689-94.

18. Fang C, Hua J, Li J, Zhen J, Wang F, Zhao Q, et al. Comparison of long-term results between laparoscopy-assisted gastrectomy and open gastrectomy with D2 lymphadenectomy for advanced gastric cancer. Am J Surg. 2014;208(3):391-6.

19. Zhang $Y$, Qi F, Jiang Y, Zhai H, Ji Y. Long-term follow-up after laparoscopic versus open distal gastrectomy for advanced gastric cancer. Int J Clin Exp Med. 2015;8(8):1356470.

20. Li Q, Wang J, Zhang G, Wang J, Yang B, Zhang Z. Feasibility and safety comparison of laparoscopy-assisted versus open gastrectomy for advanced gastric carcinoma with D2 lymphadenectomy. Jpn J Clin Oncol. 2016;46(4):323-8.

21. Hu Y, Huang C, Sun Y, Su X, Cao H, Hu J, et al. Morbidityand Mortality ofLaparoscopic Versus Open D2 Distal Gastrectomy for Advanced Gastric Cancer: A Randomized Controlled Trial. J Clin Oncol. 2016;34(12):1350-7.

22. Lin JX, Huang $\mathrm{CM}$, Zheng $\mathrm{CH}$, Li $\mathrm{P}$, Xie JW, Wang JB, et al. Is All Advanced Gastric Cancer Suitable for LaparoscopyAssisted Gastrectomy With Extended Lymphadenectomy? A Case-Control Study Using a Propensity Score Method. Ann Surg Oncol. 2016;23(4):1252-60.

23. Jeong $\mathrm{SH}$, Lee YJ, Park ST, Choi SK, Hong SC, Jung EJ, et al. Risk of recurrence after laparoscopy-assisted radical gastrectomy for gastric cancer performed by a single surgeon. Surg Endosc. 2011;25(3):872-8.
24. Chun HT, Kim KH, Kim MC, Jung GJ. Comparative study of laparoscopy-assisted versus open subtotal gastrectomy for pT2 gastric cancer. Yonsei Med J. 2012;53(5):9529.

25. Kim KH, Kim MC, Jung GJ, Choi HJ, Jang JS, Kwon HC. Comparative analysis of five-year survival results of laparoscopy-assisted gastrectomy versus open gastrectomy for advanced gastric cancer: a case-control study using a propensity score method. Dig Surg. 2012;29(2):165-71.

26. Hamabe A, Omori T, Tanaka K, Nishida T. Comparison of long-term results between laparoscopy-assisted gastrectomy and open gastrectomy with D2 lymph node dissection for advanced gastric cancer. Surg Endosc. 2012;26(6):1702-9.

27. Shinohara T, Satoh S, Kanaya S, Ishida $Y$, Taniguchi K, Isogaki J, et al. Laparoscopic versus open D2 gastrectomy for advanced gastric cancer: a retrospective cohort study. Surg Endosc. 2013;27(1):286-94.

28. Yamanaka N, Nagai E, Ohuchida K, Ueda J, Toma $\mathrm{H}$, Shimizu S, et al. Feasibility of laparoscopic gastrectomy for advanced gastric cancer with positive peritoneal cytology. Surg Today. 2013;43(8):859-64

29. Scatizzi M, Kroning KC, Lenzi E, Moraldi L, Cantafio S, Feroci F. Laparoscopic versus open distal gastrectomy for locally advanced gastric cancer: a case-control study. Updates Surg. 2011;63(1):17-23.

30. Fuchs KH. Minimally invasive surgery. Endosc. 2002;34(2):154-9.

31. Reyes CD, Weber KJ, Gagner M, Divino CM. Laparoscopic vs open gastrectomy. A retrospective review. Surg Endosc. 2001;15(9):928-31.

32. Uyama I, Sugioka A, Matsui H, Fujita J, Komori Y, Hasumi A. Laparoscopic D2 lymph node dissection for advanced gastric cancer located in the middle or lower third portion of the stomach. Gastric Cancer. 2000;3(1):505. 
33. Desiderio J, Jiang ZW, Nguyen NT, Zhang S, Reim D, Alimoglu O, et al. Robotic, laparoscopic and open surgery for gastric cancer compared on surgical, clinical and oncological outcomes: a multi-institutional chart review. A study protocol of the International study group on Minimally Invasive surgery for GASTRIc CancerIMIGASTRIC. BMJ open. 2015;5(10):e008198.

34. Gordon AC, Kojima K, Inokuchi M, Kato $\mathrm{K}$, Sugihara K. Long-term comparison of laparoscopy-assisted distal gastrectomy and open distal gastrectomy in advanced gastric cancer. Surgic Endosc. 2013;27(2):462-70.

35. Ansari D, Gianotti L, Schroder J, Andersson R. Fast-track surgery: procedure-specific aspects and future direction. Langenbecks Arch Surg. 2013;398(1):29-37.

36. Chen ZX, Liu AH, Cen Y. Fast-track program vs traditional care in surgery for gastric cancer. World J Gastroenterol. 2014;20(2):578-83.

37. Wu WC, Smith TS, Henderson WG, Eaton CB, Poses RM, Uttley G, et al. Operative blood loss, blood transfusion, and 30-day mortality in older patients after major noncardiac surgery. Ann Surg. 2010;252(1):11-7.

38. Ding J, Liao GQ, Liu HL, Liu S, Tang J. Meta-analysis of laparoscopy-assisted distal gastrectomy with D2 lymph node dissection for gastric cancer. J Surg Oncol. 2012;105(3):297-303.

39. Wang W, Li Z, Tang J, Wang M, Wang B, Xu Z. Laparoscopic versus open total gastrectomy with D2 dissection for gastric cancer: a meta-analysis. J Cancer Res Clin Oncol. 2013;139(10):1721-34.

40. Qiu J, Pankaj P, Jiang H, Zeng $\mathrm{Y}, \mathrm{Wu} \mathrm{H}$. Laparoscopy versus open distal gastrectomy for advanced gastric cancer: a systematic review and meta-analysis. Surg Laparosc Endosc Percutan Tech. 2013;23(1):1-7.

41. Huang $Y L$, Lin HG, Yang JW, Jiang FQ, Zhang $\mathrm{T}$, Yang HM, et al. Laparoscopy-assisted versus open gastrectomy with D2 lymph node dissection for advanced gastric cancer: a meta-analysis. Int J Clin Exp Med. 2014;7(6):1490-9.
42. Kim MC, Jung GJ, Kim $\mathrm{HH}$. Learning curve of laparoscopy-assisted distal gastrectomy with systemic lymphadenectomy for early gastric cancer. World J Gastroenterol. 2005;11(47):7508-11.

43. Hyung WJ, Song C, Cheong JH, Choi SH, Noh $\mathrm{SH}$. Factors influencing operation time of laparoscopy-assisted distal subtotal gastrectomy: analysis of consecutive 100 initial cases. Eur J Surg Oncol. 2007;33(3):3149.

44. Hu WG, Ma JJ, Zang L, Xue P, Xu H, Wang ML, et al. Learning curve and long-term outcomes of laparoscopy-assisted distal gastrectomy for gastric cancer. J Laparoendosc Adv Surg Tech A. 2014;24(7):487-92.

45. Kim HG, Park JH, Jeong SH, Lee YJ, Ha WS, Choi SK, et al. Totally laparoscopic distal gastrectomy after learning curve completion: comparison with laparoscopy-assisted distal gastrectomy. J Gastric Cancer. 2013;13(1):2633.

46. Zhou D, Quan Z, Wang J, Zhao M, Yang Y. Laparoscopic-assisted versus open distal gastrectomy with D2 lymph node resection for advanced gastric cancer: effect of learning curve on short-term outcomes. a meta-analysis. J Laparoendosc Adv Surg Tech A. 2014;24(3):139-50.

47. Gabriel L, Beriot-Mathiot A. Hospitalization stay and costs attributable to Clostridium difficile infection: a critical review. J Hosp Infect. 2014;88(1):12-21.

48. Lippi G, Favaloro EJ, Cervellin G. Prevention of venous thromboembolism: focus on mechanical prophylaxis. Semin Thromb Hemost. 2011;37(3):237-51.

49. Deng JY, Liang $\mathrm{H}$. Clinical significance of lymph node metastasis in gastric cancer. World J Gastroenterol. 2014;20(14):3967-75.

50. Hsu JT, Lin CJ, Sung CM, Yeh HC, Chen TH, Chen TC, et al. Prognostic significance of the number of examined lymph nodes in nodenegative gastric adenocarcinoma. Eur J Surg Oncol. 2013;39(11):1287-93.

51. Yoon SS, Yang HK. Lymphadenectomy for gastric adenocarcinoma: Should west meet east? Oncologist. 2009;14(9):871-82. 
52. de Steur WO, Dikken JL, Hartgrink HH. Lymph node dissection in resectable advanced gastric cancer. Dig Surg. 2013;30(2):96-103.

53. Quan $Y$, Huang $A$, Ye $M, X u M$, Zhuang $B$, Zhang $P$, et al. Comparison of laparoscopic versus open gastrectomy for advanced gastric cancer: an updated meta-analysis. Gastric Cancer. 2016;19(3):939-50.

54. Eberlin LS, Tibshirani RJ, Zhang J, Longacre TA, Berry GJ, Bingham DB, et al. Molecular assessment of surgical-resection margins of gastric cancer by mass-spectrometric imaging. Proc Natl Acad Sci U S A. 2014;111(7):2436-41.

55. Liang $Y$, Ding $X$, Wang $X$, Wang $B$, Deng J, Zhang $L$, et al. Prognostic value of surgical margin status in gastric cancer patients. ANZ J Surg. 2015;85(9):678-84.

56. Woo JW, Ryu KW, Park JY, Eom BW, Kim MJ, Yoon HM, et al. Prognostic impact of microscopic tumor involved resection margin in advanced gastric cancer patients after gastric resection. World J Surg. 2014;38(2):439-46.
57. Kim BS, Oh ST, Yook JH, Kim HS, Lee IS, Kim BS. Appropriate gastrectomy resection margins for early gastric carcinoma. J Surg Oncol. 2014;109(3):198-201.

58. Deng J, Zhang R, Pan Y, Ding X, Cai M, Liu $Y$, et al. Tumor size as a recommendable variable for accuracy of the prognostic prediction of gastric cancer: a retrospective analysis of 1,521 patients. Ann Surg Oncol. 2015;22(2):565-72.

59. Chen K, Xu XW, Mou YP, Pan Y, Zhou YC, Zhang RC, et al. Systematic review and meta-analysis of laparoscopic and open gastrectomy for advanced gastric cancer. World J Surg Oncol. 2013;11:182. 